\title{
A New Way To Prevent Colorectal Cancer Using Supervised Learning Technique
}

\author{
Balaji Vicharapu ${ }^{\mathrm{a}}$, Anuradha Chint ${ }^{\mathrm{b}}$, S.R. Chandra Murty Patnala ${ }^{\mathrm{c}}$ \\ ${ }^{\text {a,c }}$ Research Scholar, Department of CSE,Acharya Nagarjuna University, A.P, India \\ ${ }^{\mathrm{b}}$ Assistant professor, Department of CSE, V RSiddhartha Engineering College, India \\ av.balaji.anu@gmail.com
}

Article History: Received: 10 November 2020; Revised 12 January 2021 Accepted: 27 January 2021; Published online: 5 April 2021

\begin{abstract}
The Colorectal cancer prompts to more number of death as of late. The diagnosis of colorectal cancer as early is protected to treat the patient. To distinguish and treat this type of cancer, Colonoscopy is applied ordinarily. Several risk prediction models for colorectal cancer have been created and approved in various populations but colon cancer effecting the young adults. In this research, we projected a Supervised Learning Technique for detecting colorectal cancer in high dimensional information.One of the most important and very popular tool for performing the machine learning tasks that includesnovelty detection,classificationorregression is Support vector machine (SVM). Training the SVM requires large quantity of quadratic programming. Due to memory constraints conventional methods are not directly applied. To overcomethese inadequacies, we introduced, Least Square (LS), Particle Swarm Optimization (PSO), Quadratic Programming and Quantum-behave PSO methods for training SVM.To corroborate the competence and proficiency of our predictable system, it is developed in open source called NCSS Software.The acquiredoutcomesof these approaches are verified on a CCG1.11 Colorectal dataset and related with the particularresolution model.
\end{abstract}

Keywords: Colorectal Cancer, Machine Learning, Support Vector Machine, Particle Swam Optimization, CCG 1.11 and Classification Accuracy

\section{Introduction}

Now a days, cancer deaths is a very dangerous out of all, only $9.6 \mathrm{M}$ peoples are died due to the cancer dieses worldwide in 2018, whatever the reason/ distortion it is. In twenty five years, cancer deaths are decreased by 27 percent in the United States, but this rate is not acceptable. In 2019, more than 6, 00, 000 cancer deaths are predictable and $1.7 \mathrm{M}$ or more new cancer cases are recorded with diagnosis. "Cancer is a group of diseases in which cells in the body grow, change, and multiply out of control" [1]. In Pattern recognition domain, cancer detection is a verysignificant research area. This research paper implementing an automatic diagnostic system and classifies cancer patients by building a liner optimal classifier using support vector machine for colorectal cancer. Here four models are used for training SVM such as Quantum-behave PSO, Least Square (LS),Particle Swarm Optimization (PSO), Quadratic Programming methods and also calculated the classification accuracy. Now a day's usage of classification in medical diagnosis system gradually increases. The most important factors in diagnosis system are patient's evaluation data and experts decisions.Though, different AI techniques and classifications systems, we can minimize the classification errors those are garnered due to lack of qualified persons and also provide examination of medical information in short time and more exhaustive way. Fig1 illustrates the different steps used in classification design system. As it is outward from the remarksindicators, these steps are dependent. On the opposite, they'redepending andinterconnected, on the consequences, one may go-back to restructurepreviousphases in an effort to improve the completeoverall performance. 


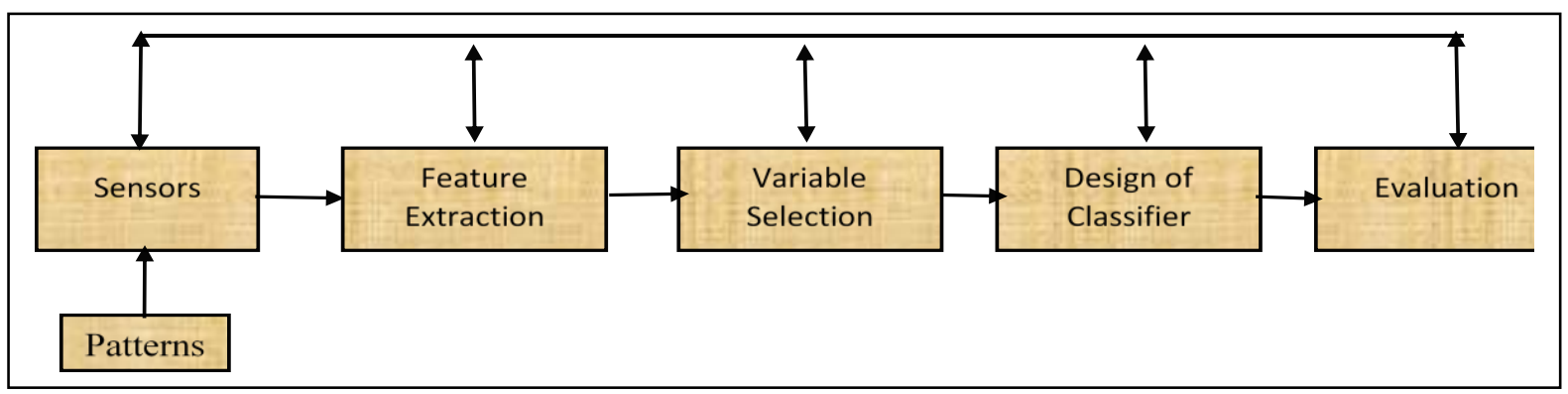

Figure 1:Basicclassification design system.

The remaining of this research work is structured as shadows. In Segment 2, the literature work relate to this field is summarized. Segment 3 examines the projected model called supervised learning system. Then, Segment 4 and 5 designates the Research Methodology in detail and compared experimental outcomes with other prototypes. In final Segment 6, summary and forthcoming work is described.

\section{Literaturereview}

In medical field, the integration and advancement of technology is rapidly increasing. Various innovative methodologies have been introduced that are helpful for identification of diseases, providing clinical trial research, radiology, drug discovery, manufacturing, personalized treatment, epidemic outbreak predictions, radiotherapy and health records etc. Various types of cancers can be detected and characterized using amount of CAD arrangements, especially it is intended/ utilized for detecting the breast tumour diseases. It is also a significant tool in the interpretation of mammographic process and support for radiologists to come into a definite conclusion. In clinic, now CAD system is utilized as second reader for recognition of breast cancer and for malignant and benign lesions classification under the advancement by many research groups. For predicting the breast cancer, many innovative techniques haven evolved in the modern days with the advancement of technology. The literature work relate to this field is summarized as follows:

Many research works from the previous studies on diagnosis and prediction of diseases is based machine learning methods for cancer recognition. Machine learning techniques includes KNN, decision trees, SVM, Bayesian classification etc. out of these classifiers KNN procedure is repeated utilized, since its adaptability and simplicity in implementation and it leads to efficient and accurateness outcomes. According to various surveys shows that KNN is most commonly used machine learning method. Liu et.al projected a prototype for cancer recognition using machine learning algorithm. Author work utilized the logistic regression model for performing the classification operation on standard breast cancer databases. Two main features called perimeter and texture are selected and accurateness of projected classifier is $96.5 \%$. Zerhouni et.al projected a prototype called Breast Cancer CAD that is based on Deep Neural networks and joint variable selection. For predicting the recurrence cutoff value, authors collect the data from Belfort hospital at France and it is named as Wisconsin Breast Cancer Database. Projected methodology is also smeared to minimize the no of response variables. The presentation of novel method increases and generates efficient and accurateness results using deep learning networks.

Bellaachia et.al projected a novel method that uses a combination of classifiers like C4.5 decision tree, the back-propagated, Naïve Bayes and neural network algorithms for breast cancer. Author uses SEER database that consists of 482,052 records and 16 attributes and this database is taken as model one due huge quantity of patient and a moderate no of attributes. Out of these projected classifiers $\mathrm{C} 4.5$ decision tree algorithm gives the better performance when compared to remaining classifiers with an accurateness of 86.7\%. A new methodology for breast cancer diagnosis was projected by Xiao et.al by combining a deep research method based machine learning feature mining processes, auto encoding method with optimal methodology for extracting the key features and information, SVM model for recognising new features into malignant tumors and benign. The projected method is tested using important breast cancer database called Wisconsin Diagnostic. Finally Experimental outcomes displays enhanced the presentation of classification and providing a capable method to breast cancer diagnosis.

Many Researchers in past years and forthcoming which are purposes to perceive the most important structures that are obliging in benevolent cancer and forecasting malignant. And also helpful for selecting the specific prototypes and selection of hyper parameters. The main aim and objective of all researchers is to generate high accurateness outcomes in less computational time.

\section{Supervised Learning System}

SVM method is widely used for classification, density estimation and regression analysis. The SVM is an accepted discriminative classifier due to its outstanding features, high accuracy and brilliant empirical throughput. 
The thought of SVM is to build a "hyper-plane" as the assessment plane in such a manner that that the edge of division between negativeand positive samples maximize as shown in figure 2 . They have been effectively applied to lots of dissimilar applications, such as text classification, speaker verification, image categorization, and bioinformatics. SVM are based on the instinctive thought of maximizing the edge of division between two challenging classes, where the border is clear as the distance between the choice hyper plane and the neighbouring training. It has been bare to be linked to minimalizingahigher bound on the interpretationfault.

For direct isolatable training pair of 2 classes, the particular verdict "hyper-plane"in multi-dimensional elementtrajectory gi(z) is known in the subsequentequivalence:

$$
\text { gi }(\mathrm{z})=\mathrm{W}_{\mathrm{i}} \mathrm{T} . \mathrm{Y}+\mathrm{w}_{\mathrm{i}} \mathrm{O}=0
$$

Anywhere $\mathrm{g}_{\mathrm{i}}(\mathrm{z})=$ Outcome feature trajectory

$\mathrm{W}_{\mathrm{i}} \mathrm{T}=\left\{\mathrm{w}_{1}, \mathrm{w}_{2} \ldots \ldots \mathrm{W}_{\mathrm{n}}\right\} \mathrm{T}=$ weight vector

$\mathrm{n}=$ Total attributes

$\mathrm{w}_{\mathrm{i}} \mathrm{O}=\mathrm{a}$ scalar verge $/$ bias weight

$\mathrm{z}=\mathrm{I} / \mathrm{p}$ feature trajectory

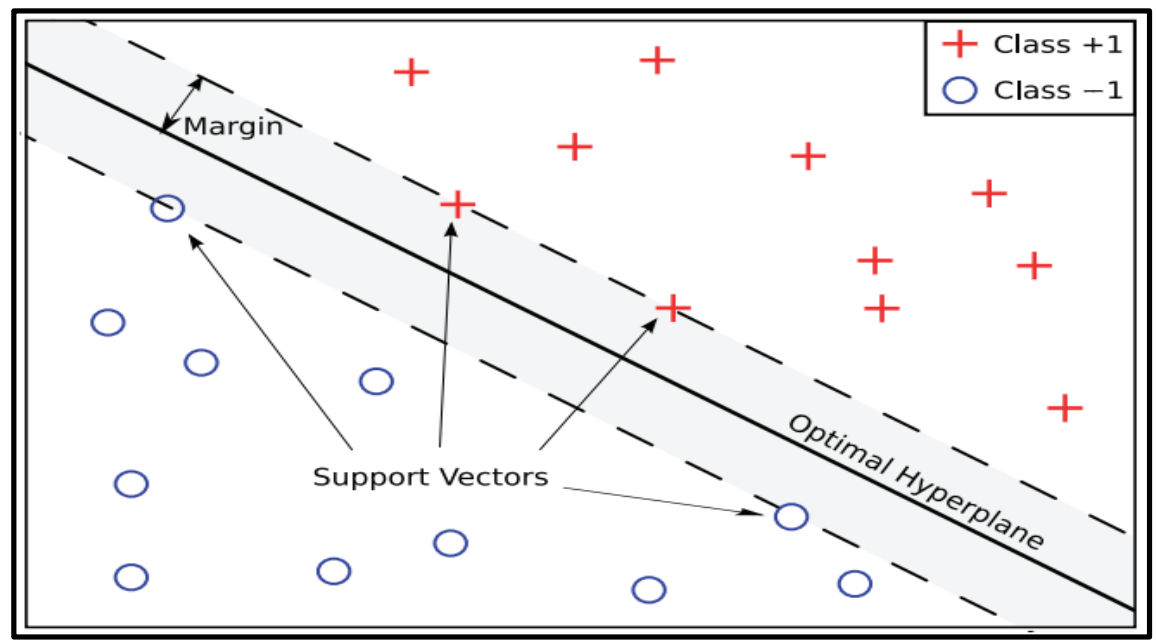

Figure 2:Maximum Margin Separations for simple classification task

The verdict"hyper-plane"then the subsequent is suitable.

$$
\begin{aligned}
& \mathrm{g}_{\mathrm{i}}\left(\mathrm{z}_{1}\right)=\mathrm{W}_{1} \mathrm{~T} \mathrm{z}_{1}+\mathrm{w}_{\mathrm{i}} 0=0 \\
& \mathrm{~g}_{\mathrm{j}}\left(\mathrm{z}_{2}\right)=\mathrm{W}_{2} \mathrm{Tz}_{2}+\mathrm{w}_{\mathrm{i}} 0=0 \ldots
\end{aligned}
$$

Subtract two equations will provide the following series of equations:

$$
\begin{aligned}
& \quad g_{i}\left(z_{1}\right)=g_{j}\left(z_{2}\right)=0 \Rightarrow W_{1} T z_{1}+w_{i} 0=W_{2} T z_{2}+w_{i} 0=0 \\
& \text { gi }\left(z_{1}\right)-g j\left(z_{2}\right)=0 \Rightarrow W_{1} T z_{1}+w i 0-W_{2} T z_{2}-w i 0=0 \\
& g_{i j}\left(z_{1}, z_{2}\right)=0 \Rightarrow W_{1} T z_{1}-W_{2} T z_{2}=0 \\
& \text { i.e. gij }\left(z_{1}, z_{2}\right)=0 \Rightarrow w T\left(z_{1}-z_{2}\right)=0 \ldots \ldots \ldots \ldots \ldots \ldots \ldots \ldots \ldots \ldots \ldots \ldots \ldots \ldots \ldots
\end{aligned}
$$

Where $\left(z_{1}-z_{2}\right)$ is a trajectory equivalent to the choice border and is intended for from $z_{1}$ to $z_{2}$. Since the pointcreation is 0 , the path for WT must be vertical to choice border. So, at all point that lies on top of the separating hyper plane.

$$
\mathrm{w} 1 \mathrm{z} 1+\mathrm{w} 2 \mathrm{z} 2+\mathrm{w}_{\mathrm{i}} 0=\mathrm{k}>0
$$

Likewise, at every point that deception underneath the sorting out "hyper-plane" fulfils i.e. for eachspherepositionedunderneath the choice border, we can display that

$\mathrm{w}_{1} \mathrm{z}_{1}+\mathrm{w}_{2} \mathrm{z}_{2}+\mathrm{w}_{\mathrm{i}} 0=0$ 
If we tag the class +1 as squares and class -1 as circles, then we can work out the class tagZ for at all test example Q1

The values can be attuned so that the hyper planes important the edges of the border can be transcribed as

$$
\mathrm{H}_{1}: \mathrm{w} 1 \mathrm{z} 1+\mathrm{w} 2 \mathrm{z} 2+\mathrm{wi} 0 \geq 1, \text { for } \mathrm{Zi}=+1
$$

$\mathrm{H}_{2}: \mathrm{w} 1 \mathrm{z} 1+\mathrm{w} 2 \mathrm{z} 2+\mathrm{wi} 0 \leq-1$, for $\mathrm{Zi}=-1$

Some tuples falls on or on top,any tuple that falls on or below $\mathrm{H}_{2}$ belongs to class -1 , and $\mathrm{H}_{1}$ fits to class +1 . Connecting the two discriminations of equivalences and we get

$\mathrm{Zi}(\mathrm{w} 1 \mathrm{z} 1+\mathrm{w} 2 \mathrm{z} 2+\mathrm{wi} 0) \geq 1$, for all $\mathrm{i}$

The edge can be computed by subtracting the statement 2 from the statement 1 . This is equal with

A scope of $\frac{1}{\|w\|}+\frac{1}{\|w\|}=\frac{2}{\|w\|}$

Necessary that

$$
\begin{aligned}
& \text { wiTz }+ \text { wio } \geq 1, \quad \forall z \in \text { w1 } \\
& \text { wiTz + wio } \leq-1, \forall z \in \text { w2 }
\end{aligned}
$$

Process the parameters w, wio of the "hyper-plane":

So, $\operatorname{diminish} \mathrm{J}(\mathrm{w}, \mathrm{wio})=\frac{1}{2}\|w\|^{2}$

$\mathrm{Zi}($ wiTzi + wio $) \geq 1, \quad \mathrm{i}=1,2$. N.

The Karush-Kuhn-Tucker expresses that the minimalize of above equivalences needs to legitimize argument:

$$
\begin{aligned}
& \frac{\partial}{\partial w} \mathrm{~L}(\mathrm{w}, \text { wio, } \chi)=0 \text { and } \frac{\partial}{\partial w o} \mathrm{~L}(\mathrm{w}, \text { wio, } \chi)=0 \text { where } \chi \mathrm{i} \geq 0 \quad \mathrm{i}=1,2 \ldots \ldots \ldots \ldots \mathrm{N} \\
& \chi_{\mathrm{i}}[\mathrm{Yi}(\text { wiTyi+wio) }-1]=0 \quad \mathrm{i}=1,2 \ldots \ldots \ldots \ldots \mathrm{N} \\
& \mathrm{L}(\mathrm{w}, \text { wio, } \chi)=\frac{1}{2} \mathrm{wTw}+\sum_{i=1}^{N} \lambda_{i}[\mathrm{Zi}(\text { wiTyi+ wio })-1]
\end{aligned}
$$

Integrate the equations (5.16), (5.17) and (5.18), we get

$$
\mathrm{W}=\sum_{i=1}^{N} \lambda_{i_{\text {Zizi }}} \text { and } \quad \sum_{i=1}^{N} \lambda_{i_{\mathrm{Zi}=0}}
$$

A novel technique based on the SVM classification for PQ disturbances. It is experimental that SVM properly classifies PQ disturbances. The projected method using SVM generates over all classification rate of $99.1 \%$. Hence the technique can be used for classification of PQ disturbances.

If two divisions are in"non-linear case", equations (10) and (11) are no longer suitable and have dissimilar procedures. The training feature vector depends on the subsequent3groups:

Trajectories that drop outer the sphere and are properly categorized. These trajectories agree with the restrictions

$\mathrm{Zi}($ wiTzi + wio $) \geq 1, \quad \mathrm{i}=1,2 \ldots \ldots \ldots \ldots . \mathrm{N}$

Trajectories lessening inside the sphere and are properly categorized. These are the arguments placed in quadrangles of the "hyper-plane" and they accomplish the discrimination

$$
0 \leq \mathrm{Zi}(\text { wiTzi }+ \text { wio })<1
$$

Trajectories that mis-classified. They are together with this by spheres and submit the discrimination

$\mathrm{Zi}($ wiTzi +wio) $<0$

Total3circumstances can be preserved under a single type of restrictions by starting a novel objective function $\varnothing$ is given by

$\mathrm{Zi}($ wiTyi + wio $) \geq 1-\varnothing \mathrm{i}$

For category Z-1: $\varnothing \mathrm{i}=0 \quad$ for category $\mathrm{Z}-2: 0 \leq \emptyset \mathrm{i}<1$ 
for categorZ-3: Øi $\geq 1$

The variables $\varnothing \mathrm{i}$ is called as slack variables. The goal now is to make the margin as giant as probable but at the similar period to retain the number of arguments with $\emptyset>0$ as slight as conceivable. This equals to adopting to minimalize the "cost-function"

$$
\mathrm{J}(\mathrm{w}, \text { wio, } \varnothing)=\frac{1}{2} \mathrm{wTw}+\mathrm{C} \sum_{i=1}^{N} I(\varnothing i)
$$

Anywhere $\varnothing$ is the trajectory of the constraints $\emptyset \mathrm{i}$ and

$$
\emptyset_{i}=1, \quad \text { if } \quad \begin{aligned}
& \varnothing i>0 ; \\
& \varnothing i=0 ;
\end{aligned}
$$

The constraint $\mathrm{C}$ is anoptimistic constant that manage the comparativeeffect of the challenging positions. Optimization issue can be resolved by minimalizing Lagrange utility.

$$
\begin{aligned}
& \mathrm{L}(\mathrm{w}, \text { wio, } \varnothing, \chi, \mathrm{u})=\frac{1}{2}\|w\|_{2+\mathrm{C}} \sum_{i=1}^{N} \varnothing i \sum_{-1=1}^{N} \varnothing i u ́ i
\end{aligned}
$$

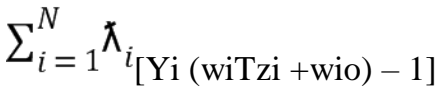

The correspondent Karush-Kuhn-Tucker circumstances that the minimalize of above equivalences has to satisfy are

$$
\begin{aligned}
& \frac{\partial L}{\partial w}=0 \text { or } \mathrm{w}=\sum_{i=1}^{N} \lambda_{i_{\text {Zizi }}} \\
& \frac{\partial L}{\partial w o}=0 \quad \text { or } \sum_{i=1}^{N} \lambda_{i} \mathrm{Zi}=0
\end{aligned}
$$

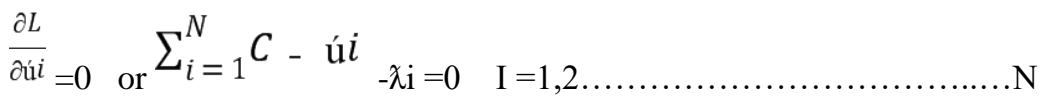

$$
\begin{aligned}
& \chi_{i}[\mathrm{Zi} \text { (wiTzi +wio) }-1+\varnothing i]=0 \text {, úiØi }=0, u ́ i \geq 0, \chi_{i} \geq 0 \quad i=1,2 \ldots \ldots . . N
\end{aligned}
$$

In non-linear circumstance, SVM compare the input trajectories y into a lofty feature space through some nonlinear comparing. In this work, the following algorithms are used to solve optimization and non-convex optimization issue.

Algorithm: SVM learning algorithm with optimal parts

Input: $\left\{\left(O^{(r)}, W_{\text {ref }}^{(r)}\right\} \mathrm{C}\right.$ and accuracy $\varepsilon$;

Initialize $\alpha$ and empty constant set: $\mathrm{Wr} \leftarrow \varnothing$

repeat

for $\mathrm{r}=1 \ldots \ldots \mathrm{R}$ do

$\left.\left.W_{\star}^{(r)} \leftarrow \arg _{w} \max _{\{\mathrm{L}(\mathrm{W},} W_{r e f}^{(r)}{ }_{+} \alpha^{T} \emptyset_{(} O^{(r)}, \mathrm{W}\right)\right\}$

if $\alpha^{T} \phi\left(O^{(r)}, W_{r e f}^{(r)}\right)<{ }_{\mathrm{L}}\left(W_{r e f}^{(r)}, W_{\star}^{(r)}\right)-E_{r_{-}} \varepsilon_{\text {then }}$

$/ *$ put it in constraint set $* /$

$$
\begin{aligned}
& \mathrm{Wr} \leftarrow \mathrm{Wr} \cup{W_{*}^{(r)} ;}_{(}^{\left.\alpha, E_{r}\right)} \leftarrow \min \frac{1}{2}\|\alpha\|_{2}^{2}{ }_{+}{ }_{R}^{C} \sum_{r=1}^{R} E_{r}
\end{aligned}
$$




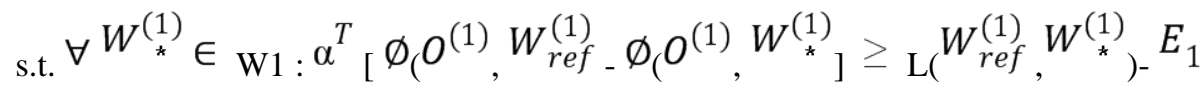

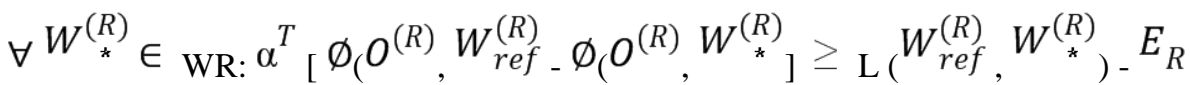

untilno Wr has changed during iteration;

return $\alpha$.

Algorithm: SVM learning algorithm with non-convex optimal segmentation

p. Initial: $\alpha=[1 ; 0 ; 0::$ : $]$ and $\theta^{(r)}=\theta_{\lambda}^{(r)}$;

1. fixing $\alpha$, optimizethe reference segmentation $\theta^{(r)}$ for every training pair

$\left.\theta_{\alpha=}^{(r)} \arg _{\theta^{(r)}}^{\max }\left\{\alpha^{T} \varnothing_{(} O^{(r)}, W_{\text {ref } ;}^{(r)} \theta^{(r)}\right)\right\}, \forall_{\mathrm{r}}$

2. Fixing $\theta_{\alpha}^{(r)}$, optimize $\alpha$ by minimalizing the subsequentcurvinghigher bound using the cutting plane procedure.

$$
\frac{1}{2}\|\alpha\|_{2}^{2}{ }_{+}^{C} \sum_{r=1}^{R}\left[-\alpha^{T} \emptyset\left(O^{(r)}, W_{r e f}^{(r)} ; \theta_{\alpha}^{(r)}\right)+\left\{\mathrm { L } \left(W_{r e f}^{(r)}, W_{)+} \alpha^{T} \emptyset\left(O^{(r)}, \mathrm{W} ; \theta\right)\right.\right.\right.
$$

3. Repeat Step 1 until congregate;

return;

\section{Svm Training Methods}

For construction of SVM classifiers, different techniques are examined. In order to determine the optimal value of nonnegative multipliers, four different methodologies (i.e. SVM training methods) are used. These methodologies include: i). Least Square Method ii). Particle Swarm Optimization iii). Quadratic programming iv).Quantum behaved PSO

\subsection{Particle swarm optimization}

In PSO, searching operation is performed via swarm of particles and updates can takes place iteration to iteration. For obtaining the optimal solution, particles are moved from previous position called pbest and hbest position in swarm. One has

$\mathrm{q}$ bes $(\mathrm{j}, t)=\arg \min \mathrm{p}=1, \ldots, t\left[\mathrm{~g}\left(\mathrm{Q}_{\mathrm{j}}(\mathrm{p})\right)\right], \mathrm{j} \in\{1,2, \ldots, \mathrm{MQ}\}$,

$\mathrm{hbes}(t)=\left[\mathrm{g}\left(\mathrm{Q}_{\mathrm{j}}(\mathrm{q})\right)\right], \arg \min \mathrm{j}=1, \ldots, \mathrm{MQp}=1$

Wherejindicates the particle index, MQ the whole quantity of particles, $t$ the current redundancyno, $\mathrm{Q}$ the positionandg the fitness function. The position Qand velocity Uof particles are rationalised by the subsequentequivalences:

$$
\begin{aligned}
& \mathrm{U}_{\mathrm{j}}(t+1)=\omega \mathrm{U}_{\mathrm{j}}(t)+\mathrm{d}_{1} \mathrm{c} 1\left(\mathrm{q} \text { bes }(\mathrm{j}, t)-\mathrm{Q}_{\mathrm{j}}(t)\right)+\mathrm{d}_{2} \mathrm{c}_{2}\left(\text { hbest }(t)-\mathrm{Q}_{\mathrm{j}}(t)\right), \\
& \mathrm{Q}_{\mathrm{j}}(t+1)=\mathrm{Q}_{\mathrm{i}}(t)+\mathrm{U}_{\mathrm{j}}(t+1), \ldots \ldots \ldots \ldots \ldots \ldots \ldots \ldots \ldots \ldots \ldots \ldots \ldots \ldots \ldots \ldots \ldots \ldots \ldots \ldots \ldots \ldots \ldots \ldots \ldots \ldots \ldots \ldots \ldots \ldots \ldots \ldots \ldots
\end{aligned}
$$

WhereU denotes the velocity, $\omega$ is the inertia weight used to balance the global search and local utilisation, $\mathrm{d}_{1}$ and $\mathrm{d}_{2}$ are optimistic constant factors called acceleration coefficients, and $r_{1}$ and $r_{2}$ are consistently scattered irregular factors inside range [0, 1]. It is common to fixahigherheaded for the speed factor. Speed packing was used as an approach to bound particles floating out of the investigation space. The 1stportion of formulation (14), known as inertia,signifies the preceding velocity, which delivers the essentialmotion for particles to travelthrough the exploration space. The 2ndportion, known as the reasoningconstituent, signifies the separate particle sophisticated of every particle. It emboldens the particles to transferto their own best placesoriginate so far. The 3rdportion, the collaborationconstituent, signifies the concertedconsequence of the particles to discovery the global optimum solution. The pseudo code representation of PSO procedure is shown below:

Stage 1. Introduction

For every particle $\mathrm{j}=1, \ldots, \mathrm{R}$, do

(a) Adjust the particle's location with a consistentlyscattering as $\mathrm{Q}(0) \sim \mathrm{U}(\mathrm{BL}, \mathrm{BU})$, 
WhereBL and BU signify the inferior and higherboundaries of the exploration space

(b) Adjustqbest to its firstplace: $q$ bes $(i, 0)=\mathrm{Q}_{\mathrm{j}}(0)$.

(c) Adjusthbest to the nominalcharge of the swarm: gbes $(0)=\operatorname{argmin} f\left[\mathrm{Q}_{\mathrm{j}}(0)\right]$.

(d) Adjustspeed: $\mathrm{U}_{\mathrm{j}} \sim(-|\mathrm{BU}-\mathrm{BL}|,|\mathrm{BU}-\mathrm{BL}|)$.

Stage 2. Replication until a endconditions is met forevery particle $\mathrm{j}=1, \ldots, \mathrm{U}$, do

(a) Élite random amounts: $c_{1}, c_{2} \sim(0,1)$.

(b) Update particle's speed. See formulation (2)

(c) Update particle's location. See formulation (3).

(d) If $\left[\mathrm{Q}_{\mathrm{j}}(t)\right]<\mathrm{g}[\mathrm{q}(\mathrm{j}, t)]$, do

(i) Update the best recognisedlocation of particle $i$ : $\mathrm{q}(i, t)=\mathrm{Q}_{\mathrm{j}}(t)$.

(ii) If $\left[\mathrm{Q}_{\mathrm{j}}(t)\right]<\mathrm{g}[\mathrm{h} b e s t(t)]$, update the swarm's best recognisedlocation: $\mathrm{h} b e s t(t)=\mathrm{Q}_{\mathrm{j}}(t)$.

(e) $t \leftarrow(t+1)$;

Stage 3. Output $\mathrm{h}(t)$ that grips the best originate solution.

\subsection{Least Square Method}

A classification problem is deliberatedas binary, taking a group oftrainingvectors (D)belongingto2 separateclasses.

$\mathrm{D}=\left\{\left(\mathrm{x}^{1}, \mathrm{y}^{1}\right) \ldots\left(\mathrm{x}^{1}, \mathrm{y}^{\mathrm{l}}\right)\right\}, \mathrm{x} \in \mathrm{Rn}, \mathrm{y} \in\{-1,+1\}$.

Wherex $\in$ Rnisanmulti-dimensionalinformationvector,witheachexamplehaving a place with both of two classes markedy $\in\{-1,+1\}$, andlis the quantity of preparing information. This examination utilizesd,c, $\varphi, \beta, \operatorname{Handr}_{\mathrm{u}}$ as info boundaries. Sox $=[\mathrm{d}, \mathrm{c}, \beta, \varphi, \mathrm{ru}, \mathrm{H}]$. inside the currentcontext of categorizing the position of the gradient, the 2 classes labelled +1 and-1maymeanstableslopeandfailedslope. TheSupport Vector Machine (SVM) approach targets building a classifier of the structure:

$\mathrm{Y}(\mathrm{x})=\operatorname{sign}\left[\sum_{k-1}^{N} \quad \alpha_{\mathrm{k}} \mathrm{y}_{\mathrm{k}} \mathrm{k}\left(\mathrm{x}, \mathrm{x}_{\mathrm{k}}\right)+\mathrm{b}\right]$

Where $\alpha_{\mathrm{k}}$ arepositiverealconstants, bisthat thescalaredge,Nisthat the quantity of the informational indexandk $\left(\mathrm{x}, \mathrm{x}_{\mathrm{k}}\right)$ istheKernelfunction.For the instance of two classes, one assumes:

$$
\begin{aligned}
& \mathrm{w}^{\mathrm{T}} \varphi\left(\mathrm{x}_{\mathrm{k}}\right)+\mathrm{b} \geq 1, \text { if } \mathrm{y}_{\mathrm{k}}=+1 \text { ( } \text { (tableslope), } \\
& \mathrm{w}^{\mathrm{T}} \varphi\left(\mathrm{x}^{\mathrm{k}}\right)+\mathrm{b} \leq 1, \text { if } \mathrm{y}_{\mathrm{k}}=-1 \text { (failed slope). }
\end{aligned}
$$

Wherewisanflexibleweightvector,Tisthat

thetranslateand $\varphi$

(.)isthat

thecomponent mapthatmapstheinputspaceintoabetterdimensionalspace, whichisequivalentto:

$\mathrm{y}_{\mathrm{k}}\left[\mathrm{w}^{\mathrm{T}} \varphi\left(\mathrm{x}^{\mathrm{k}}\right)+\mathrm{b}\right] \geq 1, \mathrm{k}=1, \ldots, \mathrm{N}$

As

indicated

by

thestructuralriskminimizationprinciple, thepossibilityboundisminimizedbyformulatingthesubsequent optimization problem:

Minimize: $1 / 2 \mathrm{w}^{\mathrm{T}} \mathrm{w}+\gamma / 2 \sum_{k=1}^{l} \quad \mathrm{e}_{\mathrm{k}}^{2}$,

Subjectedto: $\mathrm{y}_{\mathrm{k}}\left[\mathrm{w}^{\mathrm{T}} \varphi\left(\mathrm{x}_{\mathrm{k}}\right)+\mathrm{b}\right]=1-\mathrm{e}_{\mathrm{k}}, \quad \mathrm{k}=1, \ldots, \mathrm{N}$

Where $\gamma$ isthat theregularizationparameter,deciding the compromise between the fitting mistake minimizationandsmoothness, ande $\mathrm{k}_{\mathrm{k}}$ iserrorvariable.Thisoptimizationproblem(Eq.

(20))issolvedbyLagrangemultipliers, anditssolution isgivenby:

$\mathrm{Y}(\mathrm{x})=\operatorname{sign}\left[\sum_{k-1}^{N} \quad \alpha_{\mathrm{k}} \mathrm{y}_{\mathrm{k}} \mathrm{k}\left(\mathrm{x}, \mathrm{x}_{\mathrm{k}}\right)+\mathrm{b}\right]$

Wheresign ()isthat thesignumfunction.Itgives +1 (stableslope) if the component is $>=0$, and -1 (failedslope)ifitislessthanzero. 


\subsection{Quantum-behave PSO}

Heisenberg,de Broglie,Bohn, Schrödingerand Bohr are the main finding in twentieth century for the development of quantum mechanics. Their researchenforced the researchers to reconsideration the applicability of traditional mechanics and the classicalsympathetic of the surroundings of warning signs of microscopic substances. As in step with traditional PSO, a particle is indicatedvia its region trajectory $\mathrm{y}_{\mathrm{i}}$ and speedtrajectory $\mathrm{u}_{\mathrm{i}}$, which define the trajectory of the atom. The atomtransfers along a determined trajectory subsequent Newtonian mechanics. Though if we deliberate quantum mechanics, then the time period trajectory is pointless, due to the fact $\mathrm{y}_{\mathrm{i}}$ and $\mathrm{u}_{\mathrm{i}}$ of anatom cannot be determined concurrently according to uncertainty principle. Consequently, if separate particles in a PSO machine have quantum behaviour, the enactment of PSO will be distant from that of conventional PSO. In the critical model of a PSO, the nation of a particle is represented throughwave feature $\Psi(\mathrm{y}, \mathrm{t})$, in place of location and speed. The dynamic behaviour of the atom is appreciably divergent from that of the atom in conventional PSO systems. In this attitude, the likelihood of the particle's seeming in regiony from threat density feature $|\Psi(\mathrm{y}, \mathrm{t})|^{2}$, shape of which depends on the potential arena the atom lies in equation (1) shown under:

$\mathrm{y}_{\mathrm{id}}=\mathrm{y}_{\mathrm{id}}+\mathrm{u}_{\mathrm{id}}$

The particles move according to the following iterative equations:

$$
\begin{aligned}
& \mathrm{y}(\mathrm{t}+) 1=\mathrm{q}+\alpha * \text { mbest }-\mathrm{y}(\mathrm{t}) * \ln (1 / \mathrm{v}) \text { if } \mathrm{p} \geq 5.0 \\
& \mathrm{y}(\mathrm{t}+) 1=\mathrm{q}-\alpha * \text { mbest }-\mathrm{y}(\mathrm{t}) * \ln (1 / \mathrm{v}) \text { if } \mathrm{p}<5.0
\end{aligned}
$$

Where

$$
\begin{aligned}
& \mathrm{Q}=\left(\mathrm{d}_{1} \mathrm{q}_{\mathrm{id}}+\mathrm{d}_{2} \mathrm{q}_{\mathrm{gd}}\right) /\left(\mathrm{d}_{1}+\mathrm{d}_{2}\right) \ldots \ldots \ldots \ldots \ldots \ldots \ldots \ldots \ldots \ldots \ldots \ldots \ldots \ldots \ldots \ldots \ldots \ldots \ldots \ldots \\
& \text { nbest }=\frac{1}{N} \sum_{J-1}^{N} \quad \mathrm{Q}_{\mathrm{j}}=\left(\frac{1}{N} \sum_{J=1}^{N} \quad \mathrm{Q}_{\mathrm{j} 1}, \frac{1}{N} \sum_{j=1}^{N} \quad \mathrm{Q}_{\mathrm{j} 2}, \ldots \ldots \ldots \ldots\right.
\end{aligned}
$$

Mean best (mbest) of the population is particular because the average of the first-class places of all atoms, $v, p$, $\mathrm{d}_{1}$ and $\mathrm{d}_{2}$ are uniformly scattered random quantities in the interim $[0,1]$. The constraint $\alpha$ is called contractiongrowthconstant. The pseudo code representation of QPSO technique is shown under:

Step 1: Initialize the Swarm do

Step 2: Calculate nbest from the equation (5)

Step 3: Update Particle position using equation (2\&3)

Step 4: Update Q best

Step 5: Update hbest

Step 6: While maximum iteration is reached

\subsection{Quadratic programming}

The active set method is utmostcommonmethods for resolvingmedium and small scale QP problems. The idea behind the technique may be summarized as follows:

- Start with anestimate of the optimum active set $\mathrm{A}$ and compute a practicable initial iterate $\mathrm{x}_{0}$.

- Usage the Lagrange multiplier and gradient information to eliminate one key from the current active set and to add a new one. The techniqueconfirms the possibility of the next repeat $\mathrm{x}_{\mathrm{k}+1}$ designed from:

- $\quad \mathrm{x}_{\mathrm{k}+1}=\mathrm{x}_{\mathrm{k}}+\alpha_{\mathrm{k}} \mathrm{d}_{\mathrm{k}}$

Where $d_{k}$ the direction of moving and $\alpha_{k}$ is the step length, acquired by resolving a QP sub-problem. This subproblem will have a subset of restrictionsenactedas impartialities and denoted as the working set, $\mathrm{W}_{\mathrm{k}}$, containing of all $\mathrm{m}$ parityrestrictions and certain of the active discriminations. Some repeats may be positioned on the border or in the inside of the possiblearea.

- New restates are considered and the employed set is improved until the optimality circumstances are fulfilled, or all Lagrange multipliers are optimistic as needed by the KKT circumstances.

Let $\mathrm{x}_{\mathrm{k}}$ be the current iterate. At this position, some of the discriminationrestrictions may be vigorous (or satisfied as equalities). Composed with the equalityrestrictions they form the working set $\mathrm{W}_{\mathrm{e}}$ :

$$
\mathrm{Wk}=\{1, \ldots, \mathrm{m}\} \cup\{\mathrm{i}: \mathrm{a} \mathrm{T} \text { i xk }=\mathrm{bi}, \mathrm{i}=\mathrm{m}+1, \ldots, \mathrm{m}+\mathrm{p}\}
$$

For the current position, we checked whether $\mathrm{x}_{\mathrm{k}}$ reduces the quadratic objective function in the subspace definite by the working set, i.e. the Lagrange multipliers consistent to the discriminaterestrictions are positive. This is a shortestsignificance of the KKT circumstances. If the optimality circumstances are not fulfilled, we 
calculate a direction, $d_{k}$, to transfer to the next point $x_{k+1}=x_{k}+d_{k}$ such that the new repeat is practicable in $W_{k}$ and the objective function is minimalized at $\mathrm{xk}+\mathrm{dk}$. Since $\mathrm{xk}$ is recognised at the current stage, it will be observed as a continuous vector and the unidentified vector is only $\mathrm{dk}$. The problem is specified as:

$$
\min _{d k} f\left(d_{k}\right)=1 / 2\left(x_{k}+d_{k}\right)^{T} Q\left(x_{k}+d_{k}\right)+c T\left(x_{k}+d_{k}\right)
$$

Subject to:

$$
\mathrm{a}_{\mathrm{i}}^{\mathrm{T}}\left(\mathrm{x}_{\mathrm{k}}+\mathrm{d}_{\mathrm{k}}\right)=\mathrm{b}_{\mathrm{i}}, \mathrm{i} \in \mathrm{W}_{\mathrm{k}}
$$

Expanding the new objective function we have:

$$
\mathrm{f}\left(\mathrm{d}_{\mathrm{k}}\right)=1 / 2 \mathrm{x}_{\mathrm{k}}^{\mathrm{T}} \mathrm{Q} \mathrm{x}_{\mathrm{k}}+1 / 2 \mathrm{~d}_{\mathrm{k}}^{\mathrm{T}} \mathrm{Q} \mathrm{d}_{\mathrm{k}}+\mathrm{x}_{\mathrm{k}}^{\mathrm{T}} \mathrm{Q} \mathrm{d}_{\mathrm{k}}+\mathrm{c}^{\mathrm{T}} \mathrm{x}_{\mathrm{k}}+\mathrm{c}^{\mathrm{T}} \mathrm{d}_{\mathrm{k}}
$$

The term 1/ $2 \mathrm{x}_{\mathrm{k}}^{\mathrm{T}} \mathrm{Qx}_{\mathrm{k}}+\mathrm{c}^{\mathrm{T}} \mathrm{x}_{\mathrm{k}}$ is constant for a given $\mathrm{x}_{\mathrm{k}}$, thus it can be removed from the objective function without changing the solution.

We denote:

$\mathrm{g}_{\mathrm{k}}=\mathrm{Qx}_{\mathrm{k}}+\mathrm{c}$

and the function to be minimized becomes:

$\mathrm{f}(\mathrm{dk})=1 / 2 \mathrm{~d}_{\mathrm{k}}^{\mathrm{T}} \mathrm{Qd} \mathrm{d}_{\mathrm{k}}+\left(\mathrm{x}_{\mathrm{k}}^{\mathrm{T}} \mathrm{Q}+\mathrm{c}^{\mathrm{T}}\right) \mathrm{dk}=1 / 2 \mathrm{~d}_{\mathrm{k}}^{\mathrm{T}} \mathrm{Qd} \mathrm{d}_{\mathrm{k}}+1 / 2 \mathrm{~g}_{\mathrm{k}}^{\mathrm{T}} \mathrm{d}_{\mathrm{k}}$

Note that $\mathrm{Q}$ is symmetric, thus $\mathrm{Q}=\mathrm{Q}^{\mathrm{T}}$. Because $\mathrm{x}_{\mathrm{k}}$ is a feasible point within the working set $\mathrm{W}_{\mathrm{k}}$, the equivalencerestriction:

$\mathrm{a}_{\mathrm{i}}^{\mathrm{T}} \mathrm{x}_{\mathrm{k}}=\mathrm{b}_{\mathrm{i}}, \mathrm{i} \in \mathrm{W}_{\mathrm{k}}$ (8) is satisfied. From (31) and (29) we get the equivalencerestriction of the new QP sub-problem. It will be expressed as:

$\min d_{k} 1 / 2 d_{k}^{T} Q d_{k}+g_{k}^{T} d_{k}$

Subject to:

$$
\mathrm{a}_{\mathrm{i}}^{\mathrm{T}} \mathrm{d}_{\mathrm{k}}=0, \mathrm{i} \in \mathrm{W}_{\mathrm{k}}
$$

We may continue in a wayrelated to the one applied for equalityconstrained QP problems.For evaluation we try to best one technique from training proceduresstatedsuch as Subset selection processes, Iterative processes, Exploiting alternative SVM constructions.

\section{Proposed Methodology}

The following Figure 3depicts the proposed methodology for Colorectal Cancer DiagnosisModel. With this model, we can pre-process the data using scaling operation and processed data can be divided into two datasets: testing and training. SVM classifier is build using these training data and validation of each classifier is done using two important parameters: Sensitivity and Specificity in distinctive "cancer patients" from non-cancer controls. Different combination of features are used for building SVM classifies in order to reach the SVM Classifier to its maximum value. Cross validation methodology is utilized for calculating the classification accuracy and the parameter like generalization error is evaluated using validation dataset. For construction of SVM classifiers, here we used four different methodologies (i.e. SVM training methods) such as:i). Least Square Methodii). Particle Swarm Optimization iii). Quadratic programming iv).Quantum behaved PSO 


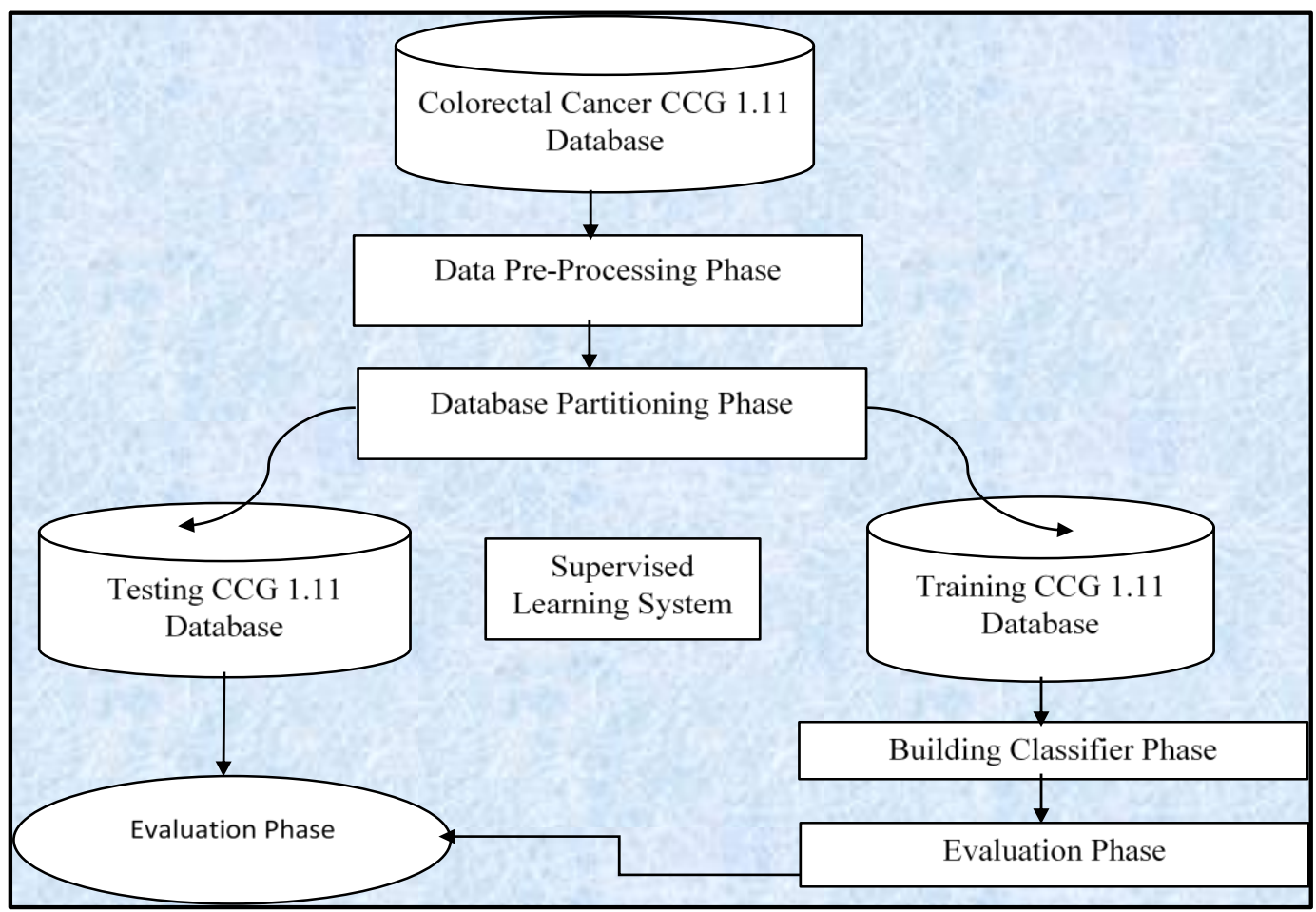

Figure 3: Proposed Methodology of Colorectal Cancer Model

The experiments are done on the Colorectal Cancer CCG 1.11 dataset from the UCl [12]. It is 1yr consistent relative subsistence proportion for adults. A cumulative pointer for 1yr subsistence for all types of cancers in adults above 15 . The probability estimation of subsistence from cancer alone is known as relative subsistence. It is definite as the proportion of the perceived subsistence and the subsistence that would have been predictable if the cancer patients had practiced the identical circumstantial humanity by sex and age as the common populace. The outcomes of the four approaches wereequated and sample CCG1.11 database is exposed in table 1:

Table 1: Sample Colorectal Cancer CCG1.11 Dataset.

\begin{tabular}{|c|c|c|c|c|c|c|}
\hline $\begin{array}{ll}\text { Year } \\
\text { diagnosis }\end{array}$ & Period of coverage & Breakdown & Level & Level Description & $\begin{array}{l}\text { Indicator } \\
\text { value }\end{array}$ & Precision \\
\hline 2011 & $\begin{array}{l}\text { Diagnosis: } 1 / 1 \text { to } 31 / 12 / 2011 \\
\text { Followed up until } 31 / 12 / 2012\end{array}$ & CCG & $00 \mathrm{C}$ & NHS Darlington CCG & 68.5 & 1.94 \\
\hline 2011 & $\begin{array}{l}\text { Diagnosis: } 1 / 1 \text { to } 31 / 12 / 2011 \\
\text { Followed up until } 31 / 12 / 2012\end{array}$ & CCG & $00 \mathrm{D}$ & $\begin{array}{l}\text { NHS Durham Dales, } \\
\text { Easington and Sedgefield } \\
\text { CCG }\end{array}$ & 67.38 & 2.8 \\
\hline 2011 & $\begin{array}{l}\text { Diagnosis: } 1 / 1 \text { to } 31 / 12 / 2011 \\
\text { Followed up until } 31 / 12 / 2012\end{array}$ & CCG & $00 \mathrm{~F}$ & NHS Gateshead CCG & 69.65 & 4.06 \\
\hline 2011 & $\begin{array}{l}\text { Diagnosis: } 1 / 1 \text { to } 31 / 12 / 2011 \\
\text { Followed up until } 31 / 12 / 2012\end{array}$ & CCG & $00 \mathrm{G}$ & $\begin{array}{l}\text { NHS Newcastle North and } \\
\text { East CCG }\end{array}$ & 70.34 & 0 \\
\hline 2011 & $\begin{array}{l}\text { Diagnosis: } 1 / 1 \text { to } 31 / 12 / 2011 \\
\text { Followed up until } 31 / 12 / 2012\end{array}$ & CCG & $00 \mathrm{H}$ & NHS Newcastle West CCG & 69.91 & 3.26 \\
\hline 2011 & $\begin{array}{l}\text { Diagnosis: } 1 / 1 \text { to } 31 / 12 / 2011 \\
\text { Followed up until } 31 / 12 / 2012\end{array}$ & CCG & $00 \mathrm{~J}$ & NHS North Durham CCG & 68 & 4.61 \\
\hline 2011 & $\begin{array}{l}\text { Diagnosis: } 1 / 1 \text { to } 31 / 12 / 2011 \\
\text { Followed up until } 31 / 12 / 2012\end{array}$ & CCG & $00 \mathrm{~K}$ & $\begin{array}{l}\text { NHS Hartlepool and } \\
\text { Stockton-On-Tees CCG }\end{array}$ & 68.53 & 3.59 \\
\hline 2011 & $\begin{array}{l}\text { Diagnosis: } 1 / 1 \text { to } 31 / 12 / 2011 \\
\text { Followed up until } 31 / 12 / 2012\end{array}$ & $\mathrm{CCG}$ & $00 \mathrm{~L}$ & NHS Northumberland CCG & 69.64 & 6.04 \\
\hline 2011 & $\begin{array}{l}\text { Diagnosis: } 1 / 1 \text { to } 31 / 12 / 2011 \\
\text { Followed up until } 31 / 12 / 2012\end{array}$ & $\mathrm{CCG}$ & $00 \mathrm{M}$ & NHS South Tees CCG & 70.37 & 5.05 \\
\hline 2011 & $\begin{array}{l}\text { Diagnosis: } 1 / 1 \text { to } 31 / 12 / 2011 \\
\text { Followed up until } 31 / 12 / 2012\end{array}$ & CCG & $00 \mathrm{~N}$ & NHS South Tyneside CCG & 69.87 & 3.8 \\
\hline
\end{tabular}

The outcomes of the four techniques were tested and equated with the above dataset called Colorectal Cancer CCG1.11.

\section{Results And Discussion}

In this segment, the efficiency of four SVN training methods are evaluated and compared. The objective of this comparison is two or more supervised learning techniques were evaluated alongside by considering the 
performance of SVM classifier (i.e. trained with PSO and Quantum) into perception. To corroborate the competence and proficiency of our predictable system, it is developed in open source called NCSS Software.In order to evaluate the efficiency of the projectedtechnique, several parameters/ measures were used. These parameters includes Error rate, negative and positive predictive values, confusion matrix, classification accuracy, specificity, sensitivity and distributed ROC curves. These measures are distributed curves (figure 4), analysis of specificity (Figure 5) and sensitivity (Figure 6), Error rate (Figure 7), classification accuracy (Figure 8), negative and positivepredictive value (Table 3 ) and confusion matrix in table 4.
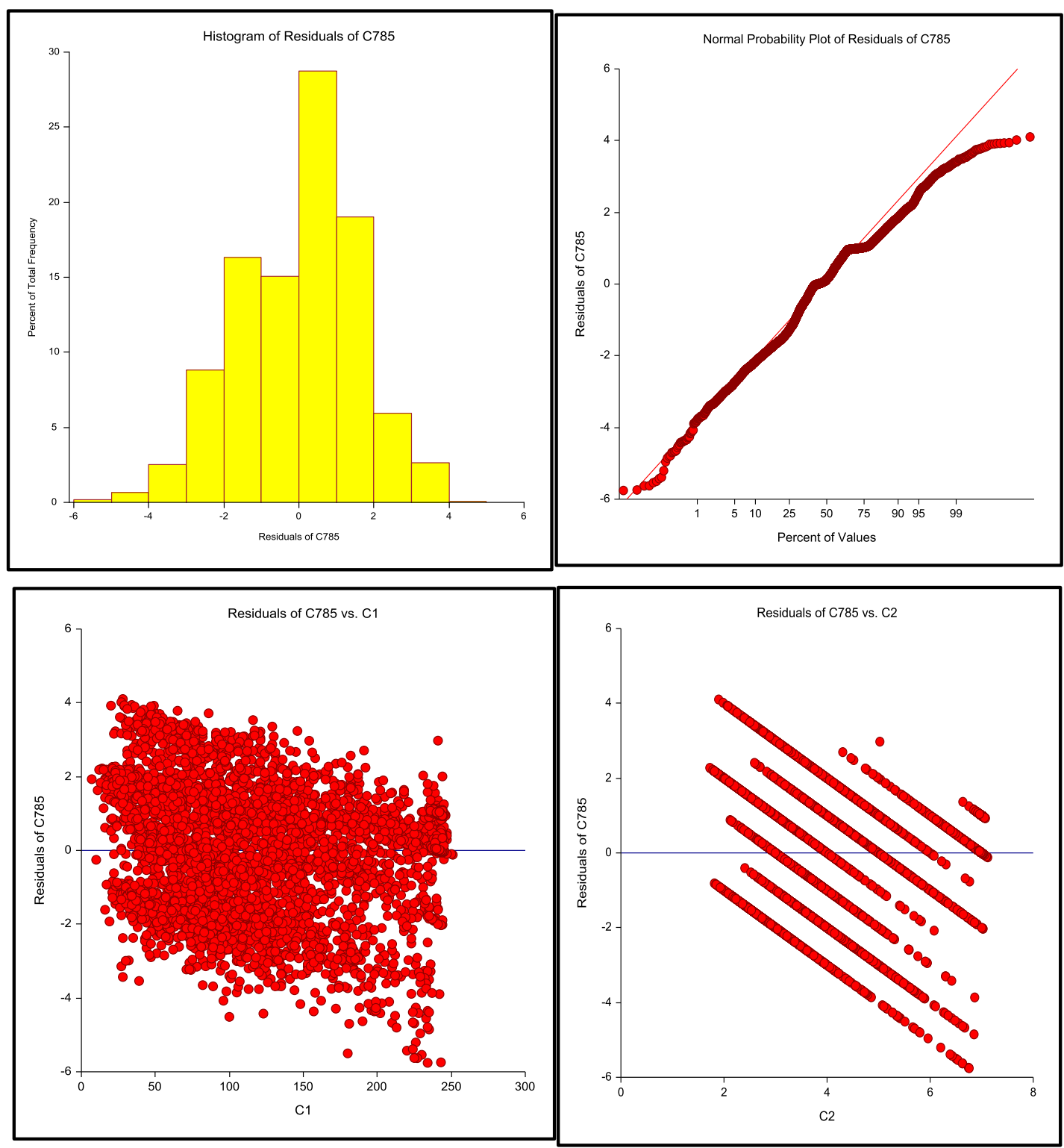

Figure 4: Distributed Curves of Colorectal Cancer CCG1.11 Dataset

Table 2:Confusion Matrix of Colorectal Cancer CCG1.11 Dataset.

\begin{tabular}{|l|l|l|l|l|l|l|}
\hline & C1 & C2 & C3 & C4 & C5 & C785 \\
\hline C1 & 1.000000 & 0.934786 & 0.831511 & 0.800765 & 0.781757 & 0.662829 \\
\hline C2 & 0.934786 & 1.000000 & 0.892806 & 0.852014 & 0.841799 & 0.687042 \\
\hline C3 & 0.831511 & 0.892806 & 1.000000 & 0.914945 & 0.834729 & 0.666610 \\
\hline C4 & 0.800765 & 0.852014 & 0.914945 & 1.000000 & 0.917275 & 0.667555 \\
\hline C5 & 0.781757 & 0.841799 & 0.834729 & 0.917275 & 1.000000 & 0.666786 \\
\hline C785 & 0.662829 & 0.687042 & 0.666610 & 0.667555 & 0.666786 & 1.000000 \\
\hline
\end{tabular}


Table 3:Comparison of SVM Training Methods with Different Parameters.

\begin{tabular}{|c|l|c|c|c|c|}
\hline \multicolumn{1}{|c|}{ S.N } & $\begin{array}{c}\text { Parameter/ Training } \\
\text { Method }\end{array}$ & PSO & $\begin{array}{c}\text { Q-B } \\
\text { PSO }\end{array}$ & QP & LSM \\
\hline 1 & Specificity & 15.996 & 15.686 & 14.620 & 15.308 \\
\hline 2 & Sensitivity & 16.185 & 16.357 & 15.480 & 15.618 \\
\hline 3 & Error rate & 04.666 & 04.987 & 11.029 & 15.635 \\
\hline 4 & PPV & 15.876 & 15.600 & 15.102 & 15.618 \\
\hline 5 & NPV & 16.271 & 16.409 & 15.067 & 15.308 \\
\hline 6 & Accuracy & 16.082 & 16.013 & 15.084 & 15.635 \\
\hline
\end{tabular}

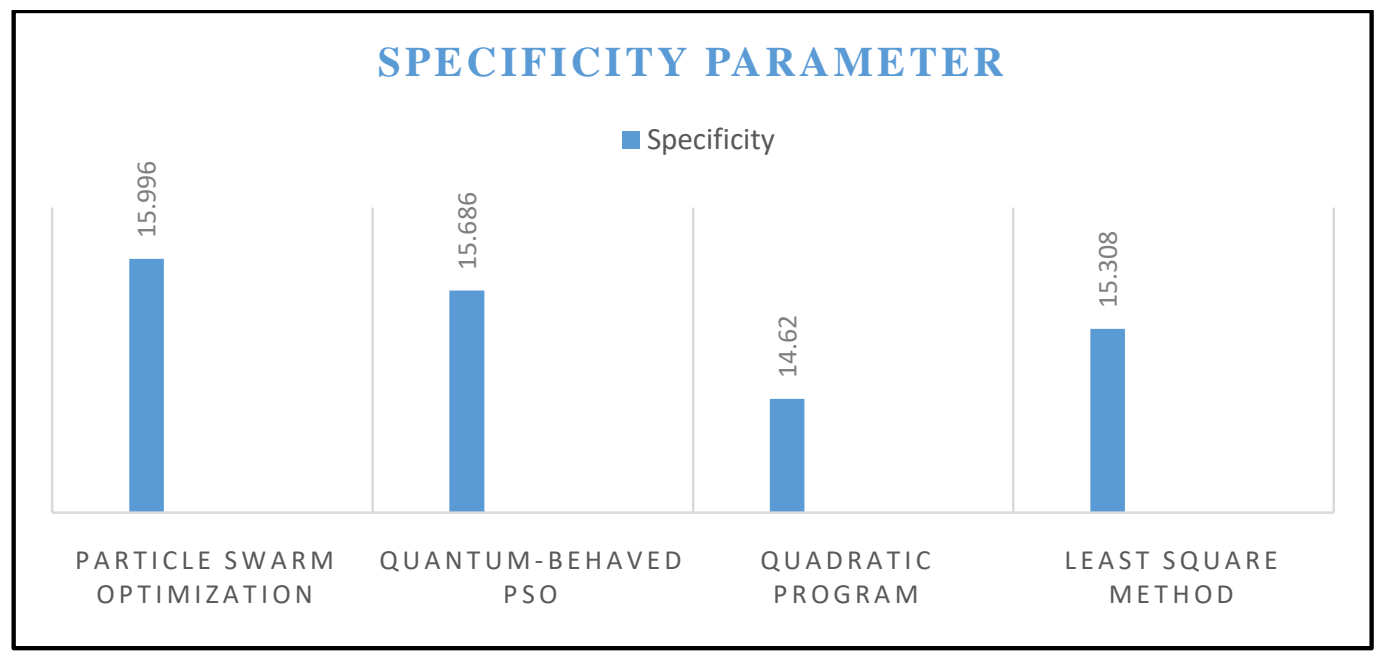

Figure 5: Specificity value onColorectal Cancer CCG1.11 Dataset. The PSO Training Method shows the highest accuracy.

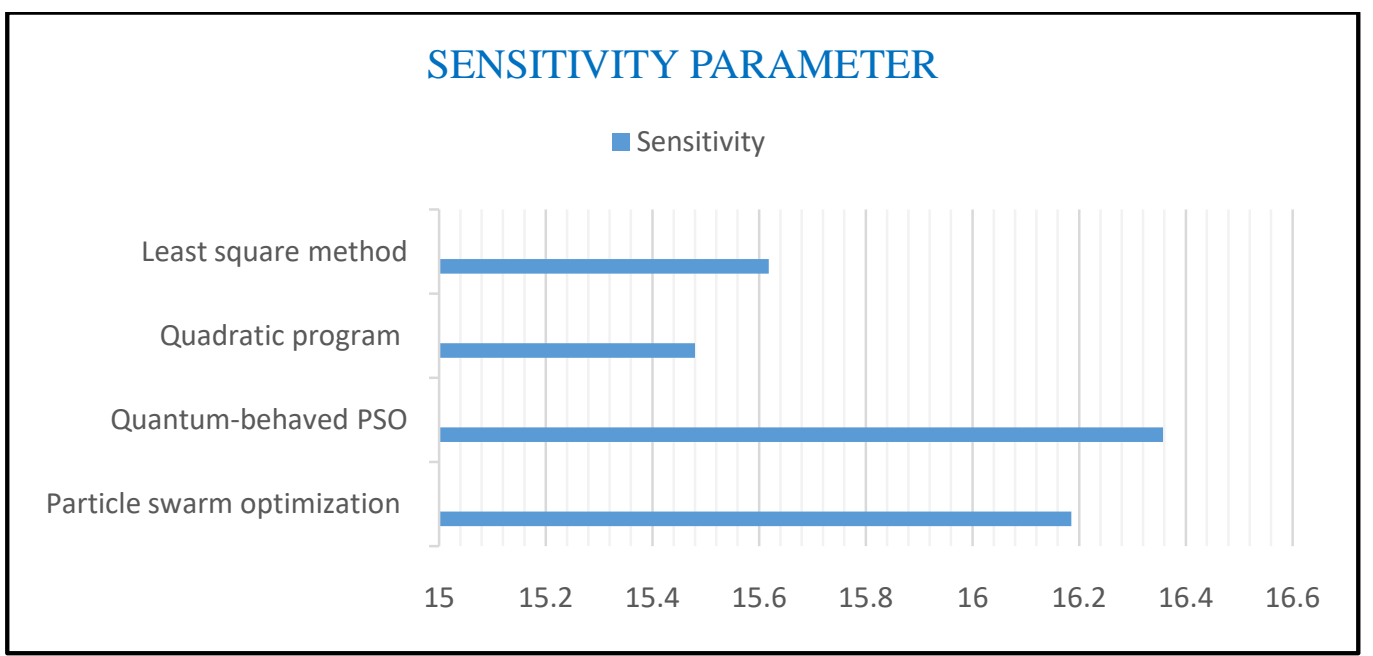

Figure 6: Sensitivity value onColorectal Cancer CCG1.11 Dataset. The QPSO Training Method shows the highest accuracy. 


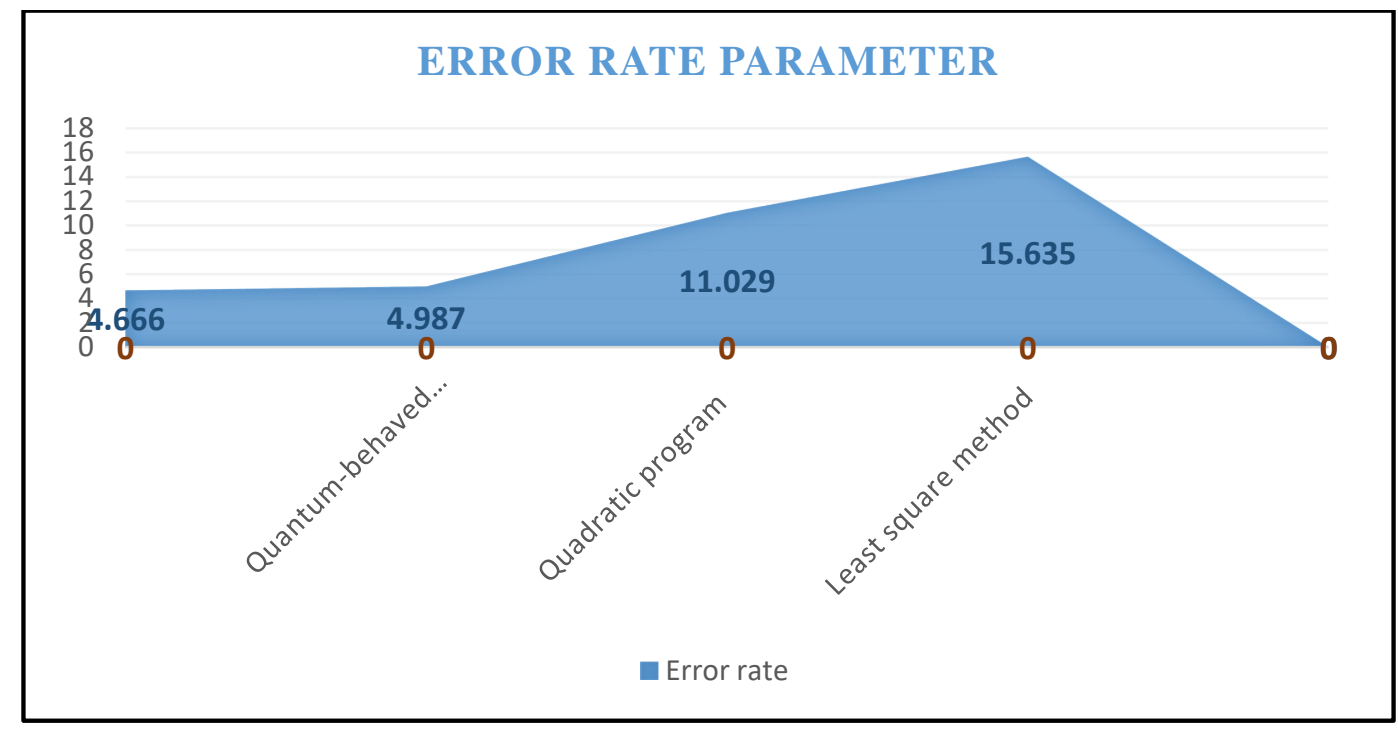

Figure 7: Error rate on Colorectal Cancer CCG1.11 Dataset. The PSO shows the lowest error.

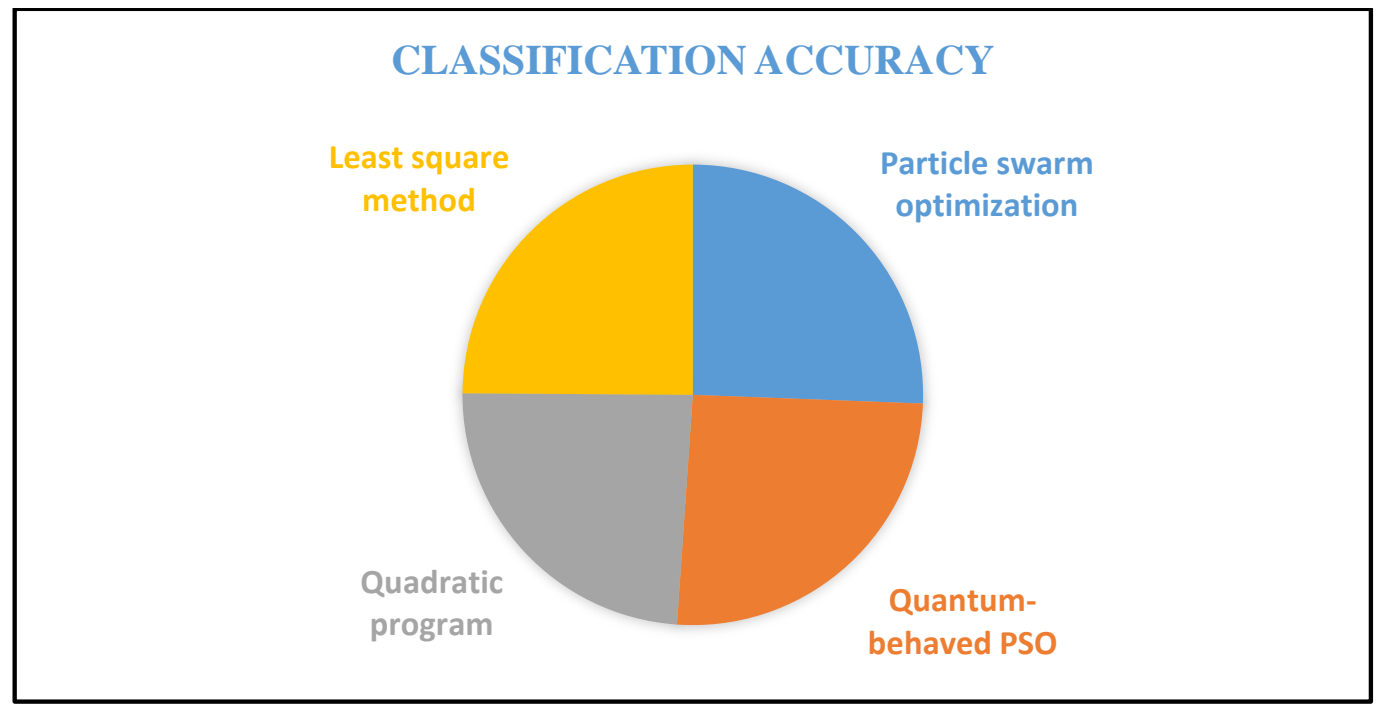

Figure 8: Correction rate value onColorectal Cancer CCG1.11 Dataset. The PSO Training Method shows the highest accuracy.

Here, we can conclude that classifier outcomes from training the SVM with Particle Swarm Optimization shows improved performance i.e. it shows best area under the curve. From ROC curve: i) the upper point $(1,1)$ represents positive classification and the point $(0,1)$ indicates perfect classification. ii) The lower point $(0,0)$ signifies no positive classification, such type of classifier obligates no false positive errors. The classifiers which are appearing LHS of ROC curve make the positive classification, which means make some false positive errors and low true positive values also. The classifiers which are appearing RHS of ROC curve make the positive classification weak evidence, which means make high false positive errors and correctly classifies all positives.

\section{Conclusion}

Colorectal cancer recognition is exact sizeable within the subject of clinical field in addition to Bioinformatics. The diagnosis of colorectal cancer as early is safe to deal with the affected person. To perceive and deal with this form of most cancers, Colonoscopy is implemented commonly. Several danger prediction models for colorectal cancer have been developed and validated in different populations but colon cancer effecting the young adults. In this research, we projected a Supervised Learning Technique for detecting colorectal cancer in high dimensional data.One of the important and very popular tool for performing the machine learning tasks that includesnovelty detection,classificationorregression is Support vector machine (SVM). Training the SVM requires large quantity of quadratic programming. Due to memory constraints conventional methods are not directly applied. To overcomethese inadequacies,we introduced, Least Square (LS), Particle Swarm Optimization (PSO), Quadratic Programming and Quantum-behave PSO methods for training SVM.To corroborate the competence and 
proficiency of our predictable system, it is developed in open source called NCSS Software.The acquired outcomesof these approaches are verified on a CCG1.11 Colorectal dataset and the classifier outcomesshows that improved performance from training the SVM with Particle Swarm Optimization

\section{References}

West, D., Mangiameli, P., Rampal, R. and West, V., 2005. Ensemble strategies for a medical diagnostic decision support system: A breast cancer diagnosis application. European Journal of Operational Research, 162(2), pp.532-551.

Liu, L., 2018, May. Research on logistic regression algorithm of breast cancer diagnose data by machine learning. In 2018 International Conference on Robots \& Intelligent System (ICRIS) (pp. 157-160). IEEE.

Yassin, N.I., Omran, S., El Houby, E.M. and Allam, H., 2018. Machine learning techniques for breast cancer computer aided diagnosis using different image modalities: A systematic review. Computer methods and programs in biomedicine, 156, pp.25-45.

Smith, J.J., Deane, N.G., Wu, F., Merchant, N.B., Zhang, B., Jiang, A., Lu, P., Johnson, J.C., Schmidt, C., Bailey, C.E. and Eschrich, S., 2010. Experimentally derived metastasis gene expression profile predicts recurrence and death in patients with colon cancer. Gastroenterology, 138(3), pp.958-968.

Janghel, R.R., Tiwari, R. and Shukla, A., 2010."Breast cancer diagnosis using artificial neural network models", inInformation Sciences and Interaction Sciences (ICIS), 2010 3rd International Conference on. IEEE, pp. 8994,

Exarchos KP, Goletsis Y, Fotiadis DI. Multiparametric decision support system for the prediction of oral cancer reoccurrence. IEEE Transactions on Information Technology in Biomedicine. 2011 Aug 18;16(6):1127-34.

Rajkumar G. Intelligent Pattern Mining and Data Clustering for Pattern Cluster Analysis using Cancer Data. International journal of Engineering Science and Technology. 2010;2(12):7459-69..

Ramaswamy, S., Tamayo, P., Rifkin, R., Mukherjee, S., Yeang, C.H., Angelo, M., Ladd, C., Reich, M., Latulippe, E., Mesirov, J.P. and Poggio, T., 2001. Multiclass cancer diagnosis using tumor gene expression signatures. Proceedings of the National Academy of Sciences, 98(26), pp.15149-15154.

Radan L, Ben-Haim S, Bar-Shalom R, Guralnik L, Israel O. The role of FDG-PET/CT in suspected recurrence of breast cancer. Cancer: Interdisciplinary International Journal of the American Cancer Society. 2006 Dec $1 ; 107(11): 2545-51$.

Kang DD, Sibille E, Kaminski N, Tseng GC. MetaQC: objective quality control and inclusion/exclusion criteria for genomic meta-analysis. Nucleic acids research. 2012 Jan 1;40(2):e15-e15.

Van Gerven M, Bohte S. Artificial neural networks as models of neural information processing. Frontiers in Computational Neuroscience. 2017 Dec 19;11:114.

Blake C. UCI repository of machine learning databases. http://www. ics. uci. edu/ mlearn/MLRepository. html. 1998 